\title{
Fractional Fourier transforms and their optical implementation: I
}

\author{
David Mendlovic \\ Faculty of Engineering, Tel Aviv University, 69978 Tel Aviv, Israel \\ Haldun M. Ozaktas \\ Department of Electrical Engineering, Bilkent University, 06533 Bilkent, Ankara, Turkey \\ Received November 30, 1992; accepted March 1, 1993

\begin{abstract}
Fourier transforms of fractional order $a$ are defined in a manner such that the common Fourier transform is a special case with order $a=1$. An optical interpretation is provided in terms of quadratic graded index media and discussed from both wave and ray viewpoints. Several mathematical properties are derived.
\end{abstract}

\section{MOTIVATION}

It is often the case that an operation originally defined for integer orders can be generalized to fractional or even complex orders in a meaningful and useful way. A basic example is the power operation. The ath power of $x$, denoted by $x^{a}$, might be defined as the number obtained by multiplying unity $a$ times with $x$. Thus $x^{3}=x \times x \times x$. This definition makes sense only when $a$ is an integer. However, it is an elementary fact that the definition of the power of a number can be meaningfully and consistently extended to real and complex values of $a$. Likewise, the original definition of the derivative of a function makes sense only for integral orders; that is, we can speak of the first or the second derivative and so on. However, it is possible to extend the definition of the derivative to noninteger orders by the use of an elementary property of Fourier transforms. Letting $\mathscr{F}[f(x)]$ denote the Fourier transform of the function $f(x)$, defined as

$$
\mathscr{F}[f(x)]=\int_{-\infty}^{+\infty} f(x) \exp \left(-i 2 \pi f_{x} x\right) \mathrm{d} x,
$$

one has the property

$$
\left(-i 2 \pi f_{x}\right)^{a} \mathscr{F}[f(x)]=\mathscr{F}\left[\frac{\mathrm{d}^{a} f(x)}{\mathrm{d} x^{a}}\right]
$$

for integer $a$. Since the left-hand side of Eq. (2) is well defined for fractional values of $a$ as well, it can be considered as the definition of the right-hand side of Eq. (2) for real $a$. Bracewell showed how fractional derivatives can be used to characterize the discontinuities of certain functions. ${ }^{1}$ [A similar definition of fractional derivatives was given long ago by Weyl (see Refs. 2 and 3). Fractional integration is defined similarly.] Based on these two examples, one is led to ask from a mathematical viewpoint whether the definition of Fourier transforms can also be extended to fractional orders.

Fourier transforms (or their generalization, Laplace transforms) play a central role in the study of linear systems. ${ }^{1}$ They are also important in modern optics because the Fourier transform arises naturally in optical systems. This fact has been the central cause for the widespread use of information processing principles in optics and of optical systems for information processing. (In fact, one often encounters the term Fourier optics being used synonymously with optical information processing.)

In an optical system involving many lenses the axial locations of the images of the input object can be determined by repeated application of familiar lens equations. Between these image planes it is possible to use the same equations to find the locations where the source would be imaged if no object were present in the system. The light distribution at these locations will have the form of the Fourier transform of the object, ${ }^{4}$ so that these planes are called Fourier-transform planes. Thus, in a system involving many lenses, one can observe the object and its Fourier transform, the object's inverted image and its Fourier transform, the object's upright image and its Fourier transform, and so on. What we will call the first Fourier transform is the common Fourier transform. For the class of functions known as self-Fourier functions, the first Fourier transform is identical to the function itself. ${ }^{5}$ The second Fourier transform is the Fourier transform of the first Fourier transform and is identical in form to $f(-x,-y)$. The fourth Fourier transform is always equal to the original function. From an optical viewpoint, one is led to inquire what is observed in such a system in the space between the images and the Fourier transforms and what is the effect of introducing spatial filters in these planes in addition to the conventional Fourier plane filter. Of course, the light distribution at any axial position can be calculated by the use of the Fresnel integral ${ }^{4}$; however, this fact is not very interesting unless it can be tied to other concepts and frameworks.

An example of a different kind of fractional operation from optics is related to the Talbot effect, ${ }^{6}$ in which self-images of an input object are observed at the twodimensional planes $z=N z_{0}$ for integer $N$ ( $z$ is the axial coordinate and $z_{0}$ is a characteristic distance). By means of a self-transformation technique ${ }^{7}$ it was shown that $N$ could also take on certain rational values. 
In Ref. 8 the authors gave an example of a fractional Fourier transform without knowing so. In this reference a self-imaging configuration containing an odd number of identical systems is suggested. If it is noted that selfimaging is a Fourier transform with order $a=4$, each of the identical systems could be considered as a fractional Fourier transformer with order $a=4 / N$, where $N$ is an odd integer. However, the authors of Ref. 8 did not notice this point.

Based on the above motivations, one might be tempted to define the functional forms of the light distributions observed in these intermediary planes as fractional Fourier transforms. We will be arguing that such conventional Fourier-transforming configurations are not a good basis for defining fractional Fourier transforms because the dual actions of focusing and propagation are not uniformly interleaved in such systems. Rather, we will base our definitions on propagation in graded index (GRIN) media. ${ }^{9}$ Such media also exhibit imaging and Fouriertransforming properties and provide a natural environment for defining fractional Fourier transforms.

In Section 2 we will introduce the notation and the basic postulates that we will require our definition to satisfy. Afterward we will discuss why GRIN media provide a natural environment for the definition of fractional Fourier transforms. Then propagation in GRIN media will be reviewed and the fractional Fourier transform defined mathematically in conjunction with its optical interpretation in GRIN media. After discussing some of the elementary properties of the fractional Fourier transform, we will examine our definition using ray optics in phase space and show that it indeed has some attractive properties.

In the sequel to this paper ${ }^{10}$ we will provide illustrative examples of fractional Fourier transforms and discuss some extensions and generalizations. We will discuss how fractional Fourier transforms can be obtained with bulk systems. Also, we will show how to generalize the spatial filtering process based on fractional Fourier transforms as well as on some computer simulations.

The fractional Fourier transform has previously been defined by mathematicians in a purely abstract way as a novel method of solving the Schrödinger equation under various conditions. ${ }^{11,12}$ Given the analogy between Maxwell's equations in GRIN media and the Schrödinger equation for the harmonic oscillator, this dual definition is not surprising. However, the authors of Refs. 11 and 12 did not mention the possibility of an optical interpretation. Note that these mathematicians did not suggest physical applications for their fractional Fourier-transform definition. Owing to the major significance of the Fourier transform in optics, we assume that this new operator, motivated by the GRIN media, will be more useful in the optics community. In Ref. 10 an application for optical signal processing is suggested. Another application for this operator could be to solve the harmonic-oscillator problem.

\section{NOTATION AND BASIC POSTULATES}

The ath Fourier transform of a function $f(x, y)$ will be denoted as $\mathscr{F}^{a}[f(x, y)]$ or simply as $F^{a} a$ when there is no confusion. We require that our definition satisfy two basic postulates. First, $\mathscr{F}^{1} f$ should be the usual first Fourier transform (the Fourier property), defined as

$$
\left(\mathscr{F}^{1} f\right)\left(x^{\prime}, y^{\prime}\right)=\int_{-\infty}^{+\infty} \int_{-\infty}^{+\infty} f(x, y) \exp \left[\frac{-i 2 \pi\left(x^{\prime} x+y^{\prime} y\right)}{s^{2}}\right] \mathrm{d} x \mathrm{~d} y,
$$

where $s, x, x^{\prime}, y$, and $y^{\prime}$ all have the dimensions of length. In a conventional $2 f$ optical Fourier-transforming configuration ${ }^{13}(x, y)$ would denote the coordinates of the input plane, $\left(x^{\prime}, y^{\prime}\right)$ would denote the coordinates of the Fourier plane, and $s^{2}=\lambda f(\lambda$ is the wavelength of light and $f$ is the focal length of the lens). The parentheses on the lefthand side of Eq. (3) emphasize that the variables $\left(x^{\prime}, y^{\prime}\right)$ belong to the function $\left(\mathscr{F}^{1} f\right)$ and not $f$.

Our second postulate requires that

$$
\mathscr{F}^{a}\left[\mathscr{F}^{b} f\right]=\mathscr{F}^{a} \mathscr{F}^{b} f=\mathscr{F}^{b} \mathscr{F}^{a} f=\mathscr{F}^{a+b} f ;
$$

that is, repeated applications of the fractional Fourier transform operator $\mathscr{F}^{a}$ should be additive (the semigroup property).

\section{GRADED INDEX MEDIA AS A BASIS FOR DEFINING FRACTIONAL FOURIER TRANSFORMS}

GRIN media will play a central role in our definition of fractional Fourier transforms. To motivate their use, we first discuss them as the limiting case of a cascade of a larger and larger number of weaker and weaker focusing systems.

Let us begin by recalling that the common optical Fourier-transform operation has two ingredients, freespace propagation and focusing by a positive lens. We can speak of these operations as being duals or Fourier conjugates of each other.,14 Indeed, as discussed in Ref. 4, the effects of propagation and focusing on an incident light distribution can be described by convolution and multiplication, respectively, by functions of the same form,

$$
\exp \left[-i \pi\left(x^{2}+y^{2}\right) / C\right]
$$

where $C=\lambda \Delta z$ for propagation over a distance $\Delta z$ and $C=-\lambda f$ for focusing by a lens of focal length $f$.

In a conventional $2 f$ Fourier-transforming configuration the act of focusing is fully concentrated at the lens and segregated from the act of propagation. We can define $\mathscr{F}^{1 / 2} f$ by distributing the focusing through the propagation as shown in Fig. 1. The distance $l$ and the focal power $f_{1 / 2}$ are chosen so that two such systems in cascade yield the first Fourier transform of the input. One can find that, for any arbitrary length $f_{1}$, the two free parameters of this configuration are

$$
f_{1 / 2}=f_{1} / \sin (\pi / 4), \quad l=f_{1} \tan (\pi / 8) .
$$

Consistent with our two postulates, we can now extend this argument to define $\mathscr{F}_{\mathcal{F}}^{1 / Q}[\cdot]$ for integer $Q$ as that operation which, when applied $Q$ times, gives the first (conventional) Fourier transform of $f$. An optical system that performs this operation may be realized by the insertion of a lens of appropriate focal length midway between the 


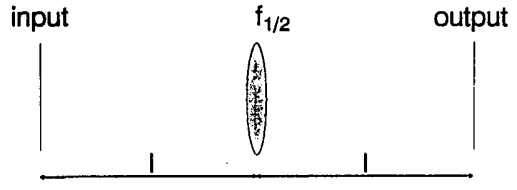

Fig. 1. Optical setup for defining the $\mathscr{F}^{1 / 2}$ operator.

appropriately spaced input and output planes, as in Fig. 1. Then $f_{1 / Q}$ and $l$ become

$$
f_{1 / Q}=\frac{f_{1}}{\sin (\pi / 2 Q)}, \quad l=f_{1} \tan \left(\frac{\pi}{4 Q}\right),
$$

respectively.

It is now possible to define Fourier transforms of rational order $\mathscr{F}^{P / Q} f$ by repeated application ( $P$ times) of the operator $\mathscr{F}^{1 / Q}$. The definition can be generalized to real orders by a limiting process.

To repeat, in a conventional $2 f$ system the focusing action is concentrated at the lens location. The same operation can also be performed by $Q$ fractional Fouriertransform stages in cascade, each performing $\mathscr{F}^{1 / Q}[\cdot]$. Note that the larger $Q$ is, the more evenly distributed the focusing action becomes and the less segregated it becomes from the propagation action. In the limit $Q \rightarrow \infty$ focusing and propagation will be infinitesimally and uniformly interspersed between each other. Of course, bulk systems with even moderately large $Q$ would be quite impractical. Fortunately, systems satisfying the same property can be realized as quadratic GRIN media. Such media can be regarded as consisting of infinitesimal layers in which focusing and propagation take place simultaneously. This property is apparent on examination of the refractive index distribution of such media, ${ }^{15}$

$$
n^{2}(r)=n_{1}^{2}\left[1-\left(n_{2} / n_{1}\right) r^{2}\right],
$$

where $r^{2}=x^{2}+y^{2}$ is the radial distance from the optical axis and $n_{1}, n_{2}$ are the GRIN medium parameters. Figure 2 shows a typical refractive index profile of such a quadratic GRIN medium. As is commonly done, we assume that the field distributions of interest are confined to a neighborhood of the optical axis such that the value of $n(r)$ dictated by the above equation is $\geq 1$. By solving the ray equation, one can show ${ }^{15}$ that a parallel bundle of rays will be focused a distance $L \equiv(\pi / 2)\left(n_{1} / n_{2}\right)^{1 / 2}$ from the input plane. In Section 6 we show that if a function $f(x, y)$ is presented at the input plane $z=0$, then at the plane $z=L$ we observe $\mathscr{F}^{1} f$ as given by Eq. (3). In other words, the focusing property of GRIN media is confirmed from a wave optics viewpoint as well. Now, since the system is fully uniform in the axial direction, $\mathscr{F}^{a} f$ can be physically defined as the functional form of the scalar light distribution at $z=a L$. Note that this definition would not be relevant in a bulk system, since the focusing and the propagation are not uniformly interleaved in that case.

Above we have motivated and defined the fractional Fourier transform in physical terms. However, it is important to note that fractional Fourier transforms can be defined purely mathematically (as in Refs. 11 and 12) and GRIN media introduced afterward as a physical interpretation. The mathematical definition will be presented in the following sections, along with its connection to propa- gation in GRIN media. We will return to discuss bulk systems in greater detail in the sequel to this paper. ${ }^{10}$

Although we cannot exclude the possibility of other definitions consistent with our two postulates, our particular definition is seen to be a natural and meaningful one, especially in an optical context.

\section{REVIEW OF PROPAGATION IN GRADED INDEX MEDIA}

The self-modes of circular quadratic GRIN media are the members of the Hermite-Gaussian (HG) function set. All the members of this self-mode set are mutually orthogonal. This function set is also an infinite and orthogonal solution set of the Schrödinger equation for the harmonic oscillator. ${ }^{15,16}$ As such, it is also complete.

The HG functions have the form

$$
\Psi_{l m}(x, y)=H_{l}\left(\frac{\sqrt{2} x}{\omega}\right) H_{m}\left(\frac{\sqrt{2} y}{\omega}\right) \exp \left(-\frac{x^{2}+y^{2}}{\omega^{2}}\right)
$$

where $H_{l}$ and $H_{m}$ are Hermite polynomials of orders $l$ and $m$, respectively,

$$
\omega=(2 / k)^{1 / 2}\left(n_{1} / n_{2}\right)^{1 / 4},
$$

with $k=2 \pi n_{1} / \lambda$, and $\lambda$ is the wavelength. Each HG mode propagates through the GRIN medium with a different propagation constant ${ }^{15}$

$$
\begin{aligned}
\beta_{l m} & =k\left[1-\frac{2}{k}\left(\frac{n_{2}}{n_{1}}\right)^{1 / 2}(l+m+1)\right]^{1 / 2} \\
& \approx k-\left(\frac{n_{2}}{n_{1}}\right)^{1 / 2}(l+m+1) .
\end{aligned}
$$

As a result of Eq. (9) and relation (11) the field distribution of a specific mode $(l, m)$ that propagates through the medium can be written as

$$
E_{l m}(x, y, z)=\Psi_{l m}(x, y) \exp \left(i \beta_{l m} z\right) .
$$

\section{FORMAL DEFINITION OF FRACTIONAL FOURIER TRANSFORMS}

Because of the orthogonality and the completeness of the HG function set, every two-dimensional function $f(x, y)$ can be expressed as

$$
\begin{aligned}
f(x, y) & =\sum_{l} \sum_{m} A_{l m} \Psi_{l m}(x, y) \\
A_{l m} & =\int_{-\infty}^{\infty} \int_{-\infty}^{\infty} f(x, y) \frac{\Psi_{l m}(x, y)}{h_{l m}} \mathrm{~d} x \mathrm{~d} y
\end{aligned}
$$

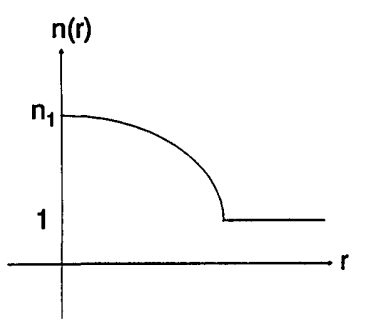

Fig. 2. Typical refractive index profile of a quadratic GRIN medium. 
where

$$
h_{l m}=2^{l+m} l ! m ! \pi \omega^{2} / 2 .
$$

Now the fractional Fourier transform of $f(x, y)$ of order $a$ can be defined as

$$
\mathscr{F}^{a}[f(x, y)]=\sum_{l} \sum_{m} A_{l m} \Psi_{l m}(x, y) \exp \left(i \beta_{l m} a L\right)
$$

As discussed in Section 2, we require that our fractional Fourier-transform definition satisfy two postulates, the Fourier [Eq. (3)] and semigroup [Eq. (4)] properties. The second can be proved directly from Eq. (16). Let us apply the operator $\mathscr{F}^{b}$ to the right-hand side of Eq. (16):

$$
\begin{aligned}
\mathscr{F}^{b}\left[\mathscr{F}^{a} f\right] & =\mathscr{F}^{b}\left[\sum_{l} \sum_{m} A_{l m} \Psi_{l m}(x, y) \exp \left(i \beta_{l m} a L\right)\right] \\
& =\sum_{l} \sum_{m} A_{l m} \Psi_{l m}(x, y) \exp \left[i \beta_{l m}(a+b) L\right]=\mathscr{F}^{a+b} f .
\end{aligned}
$$

The more complicated proof of the Fourier property is given in Section 6. In this proof we mathematically confirm that the $a=1$ st Fourier transform, as defined above, is indeed equivalent to the standard definition given in Eq. (3), provided that we choose the scale factor $s$ [see Eq. (3)], where

$$
s=\omega \sqrt{\pi}
$$

Readers who are willing to accept the above results on faith may skip to Section 8.

\section{PROOF OF THE SEMIGROUP PROPERTY}

In this section we prove Eq. (3) for the fractional Fouriertransform definition of Section 5. For the sake of notational compactness, we present the proof for onedimensional functions. The extension to two dimensions is straightforward.

The strategy of the following proof is to calculate the right-hand side of Eq. (3) for each HG function and to compare the result with the left-hand side with the use of definition (16).

The Hermite polynomials could be calculated with ${ }^{17}$

$$
H_{n}(x)=(-1)^{n} \exp \left(x^{2}\right) \frac{\mathrm{d}^{n}}{\mathrm{~d} x^{n}} \exp \left(-x^{2}\right)
$$

which leads to the following lower-order polynomials:

$$
H_{0}(x)=1, \quad H_{1}(x)=2 x, \quad H_{2}(x)=4 x^{2}-2 .
$$

A recursive definition of the Hermite polynomials is ${ }^{18}$

$$
H_{n+1}(x)=2 x H_{n}(x)-2 n H_{n-1}(x),
$$

or $^{19}$

$$
\frac{\mathrm{d}}{\mathrm{d} x} H_{n}(x)=2 n H_{n-1}(x) \text {. }
$$

The following proof uses the induction technique over $n$. That is, we first demonstrate that our hypothesis holds for $n=0$ and $n=1$; then we assume that it holds for $n-1$ and $n$ and show that this assumption implies that the hypothesis holds for $n+1$. Our hypothesis is that

$$
\mathscr{F}^{1}\left[\Psi_{n}\left(x^{\prime}\right)\right]=\sqrt{\pi} \omega \Psi_{n}(x)(-i)^{n} .
$$

Let us check it for $n=0$ :

$$
\mathscr{F}^{1}\left[\Psi_{0}\left(x^{\prime}\right)\right]=\int_{-\infty}^{\infty} \exp \left(-\frac{x^{\prime 2}}{\omega^{2}}-\frac{i 2 \pi x x^{\prime}}{s^{2}}\right) \mathrm{d} x^{\prime} .
$$

With the well-known mathematical integral ${ }^{19}$

$$
\int_{-\infty}^{\infty} \exp \left(-p^{2} x^{2} \pm q x\right) \mathrm{d} x=\exp \left(\frac{q^{2}}{4 p^{2}}\right)\left(\frac{\sqrt{\pi}}{p}\right),
$$

we see that Eq. (24) follows exactly the induction assumption (23). Since the recursive definition [Eq. (21)] depends on two previous orders, we must demonstrate that our hypothesis also holds for $n=1$. This demonstration can be performed in a similar way with the use of the integral ${ }^{19}$

$$
\int_{-\infty}^{\infty} x \exp \left(-p x^{2}+2 q x\right) \mathrm{d} x=\left(\frac{q}{p}\right)\left(\frac{\pi}{p}\right)^{1 / 2} \exp \left(\frac{q^{2}}{p}\right)
$$

Now we assume that our hypothesis holds for $n-1$ and $n$ and show that this assumption implies that the hypothesis holds for $n+1$. For this purpose we use the recursive definitions (21) and (22). Finally, we obtain

$$
\mathscr{F}^{1}\left[\Psi_{n+1}\left(x^{\prime}\right)\right]=(-i)^{n+1} \sqrt{\pi} \omega \Psi_{n+1}(x),
$$

exactly as our hypothesis predicts.

For the sake of comparison with Eq. (16), we can rewrite Eq. (23) as

$$
\mathscr{F}^{1}\left[\Psi_{n}\left(x^{\prime}\right)\right]=\Psi_{n}(x) \sqrt{\pi} \omega \exp (-i n \pi / 2) .
$$

Comparing Eqs. (16) and (28), we observe that

$$
L=(2 \pi / 4)\left(n_{1} / n_{2}\right)^{1 / 2}
$$

which is exactly the focal length that is obtained with ray optics. $^{15}$

\section{BASIC PROPERTIES}

In this section some of the mathematical properties of the fractional Fourier-transform operator are investigated.

\section{Linearity}

Let us denote two original functions by the lowercase letters $f(x)$ and $g(x)$. If $\mathscr{F}^{a} f(x)$ and $\mathscr{F}^{a} g(x)$ are the respective fractional Fourier transforms, then

$$
\mathscr{F}^{a}\left[c_{1} f(x)+c_{2} g(x)\right]=c_{1} \mathscr{F}^{a} f(x)+c_{2} \mathscr{F}^{a} g(x),
$$

where $c_{1}$ and $c_{2}$ are complex constants.

The proof of this property is based on defining the HG components of $f(x)$ and $g(x)$, namely, $A_{n}^{f}$ and $A_{n}^{8}$, respectively, such that

$$
f(x)=\sum_{n} A_{n}^{f} \Psi_{n}(x), \quad g(x)=\sum_{n}^{n} A_{n}^{g} \Psi_{n}(x) .
$$


Because of the linearity of the integral operation, one can notice that the HG components of $c_{1} f+c_{2} g$ are $c_{1} A_{n}^{f}+$ $c_{2} A_{n}^{g}$, and thus

$$
c_{1} f(x)+c_{2} g(x)=\sum_{n}\left(c_{1} A_{n}^{f}+c_{2} A_{n}^{g}\right) \Psi_{n}(x),
$$

which leads directly to Eq. (30).

\section{Continuity}

For two fractional Fourier-transform operations with orders $a_{1}$ and $a_{2}$, we can write

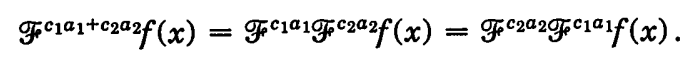

The proof makes use of Eq. (16):

$$
\begin{aligned}
\mathscr{F F}^{c_{1} a_{1}+c_{2} a_{2}} f(x) & =\sum_{n} A_{n}^{f} \Psi_{n}(x) \exp \left[-i \beta_{n} L\left(c_{1} a_{1}+c_{2} a_{2}\right)\right] \\
& =\sum_{n} A_{n}^{f} \exp \left(-i \beta_{n} L c_{1} a_{1}\right) \exp \left(-i \beta_{n} L c_{2} a_{2}\right) \\
& =\mathscr{F}^{c_{1} a_{1}}\left[\sum_{n} A_{n}^{f} \exp \left(-i \beta_{n} L c_{2} a_{2}\right) \Psi_{n}(x)\right] \\
& =\mathscr{F}_{1} c_{1} a_{1} \mathscr{F}_{2} c_{2} a_{2} f(x) .
\end{aligned}
$$

A similar procedure can be followed for proving the second equality in Eq. (33).

\section{Self-Imaging}

An interesting special case of Eq. (33) arises when $c_{2} a_{2}=$ 4. Then

$$
\mathscr{F F}^{c_{1} a_{1}+4} f(x)=\mathscr{F F}^{c_{1} a_{1} \mathscr{F}^{4}} f(x)=\mathscr{F F}^{c_{1} a_{1}} f(x) .
$$

That is, the above condition leads to self-imaging of the transformed plane. Note that for self-Fourier functions (see Ref. 7) self-imaging is also obtained for $c_{2} a_{2}=1$.

\section{Partial Convolution/Correlation}

A possible definition for the convolution operation of $f(x)$ and $g(x)$ is

$$
\operatorname{CONV}(f, g)=\mathscr{F}^{-1}\left[\mathscr{F}^{1} f(x) \times \mathscr{F}^{1} g(x)\right] .
$$

Let us denote this operation as the $a=1$ st convolution. Based on the fractional Fourier-transform definition, a convolution with the fractional order $a$ is defined as

$$
\operatorname{CONV}^{a}(f, g)=\mathscr{F}^{-a}\left[\mathscr{F}^{a} f(x) \times \mathscr{F}^{a} g(x)\right] .
$$

The fractional correlation is similarly defined as

$$
\operatorname{CORR}^{a}[f(x), g(x)]=\operatorname{CONV}^{a}\left[f(x), g^{*}(-x)\right] .
$$

\section{Additional Properties}

In their papers Namias ${ }^{11}$ and McBride and Kerr ${ }^{12}$ obtained some additional properties of the fractional Fouriertransform operation. These results are given below without their mathematical proofs:

\section{Multiplication Rule}

$\mathscr{F}^{a}\left[y^{m} f(y)\right](x)=[x \cos (2 \pi a)-i \sin (2 \pi a) D]^{m \mathscr{F}^{a}}[f(y)](x)$,

where $D$ denotes the differential operator $\mathrm{d} / \mathrm{d} x$.

\section{Differentiation Rule}

$$
\begin{aligned}
\mathscr{F F}^{a}\left[D^{m} f(y)\right](x) \\
\quad=[-i x \sin (2 \pi a)+\cos (2 \pi a) D]^{m \mathscr{F}^{a}}[f(y)](x) .
\end{aligned}
$$

Mixed Product Rule

$$
\begin{aligned}
\mathscr{F}^{a}\left[(y D)^{m} f(y)\right](x)= & \left\{-\left[\sin (2 \pi a)+i x^{2} \cos (2 \pi a)\right] \sin (2 \pi a)\right. \\
& +x \cos (2 \pi a) D-i \sin (2 \pi a) \\
& \left.\times \cos (2 \pi a) D^{2}\right\}^{m} \mathscr{F}^{a}[f(y)](x)
\end{aligned}
$$

\section{Shift Rule}

$$
\begin{aligned}
\mathscr{F}^{a} \mathscr{T}^{b}[f(y)](x)= & \exp (-i b \sin \{2 \pi a[x+0.5 b \cos (2 \pi a)]\}) \\
& \times \mathscr{F}^{a}\{f(y)\}[x+b \cos (2 \pi a)],
\end{aligned}
$$

where $\mathscr{T}^{b}$ denotes the shift operator:

$$
\mathscr{T}^{b}[f](x)=f(x+b) .
$$

One can notice from this rule that the intensity of the transformed shifted function is always the same; in other words, the intensity is shift invariant.

\section{Exponential Rule}

$$
\begin{aligned}
\mathscr{F}^{a}\{\exp (i b y) f(y)\}(x)= & \exp (i b \cos \{2 \pi a[x+0.5 b \sin (2 \pi a)]\}) \\
& \times \mathscr{F}^{a}\{f(y)\}[x+b \sin (2 \pi a)] .
\end{aligned}
$$

\section{RAY-OPTICS INTERPRETATION}

We will now examine the behavior of optical fractional Fourier transforms from a ray-optics viewpoint. In doing so, we will make use of a phase-space representation, which is also closely related to the well-known matrix formulation of ray optics. ${ }^{18}$

Let a particular paraxial ray be characterized by its radial distance $r$ and slope $s$, both with respect to the optical axis at a particular axial position $z$. Then the effect of passing through any optical system on this ray can be described as a movement in $(r, s)$ space. For instance, free-space propagation over a length $\Delta z$ corresponds to a horizontal displacement $\Delta r$ given by [Fig. 3(a)]

$$
\Delta r=r(z+\Delta z)-r(z)=s(z) \Delta z .
$$

On the other hand, focusing by a lens of focal length $f$ corresponds to a vertical displacement by [Fig. 3(b)]

$$
\Delta s=s(z+\Delta z)-s(z)=-r(z) / f .
$$
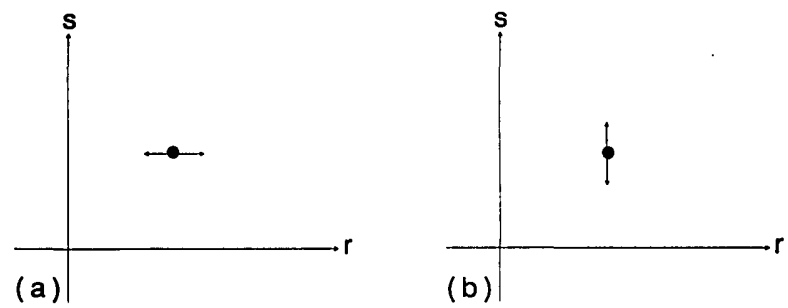

Fig. 3. Effect of (a) propagation and (b) focusing by a lens on a given ray in $(r, s)$ space. 


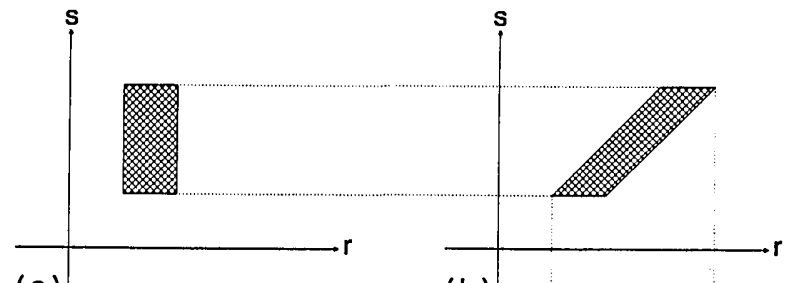

(a)

(b)

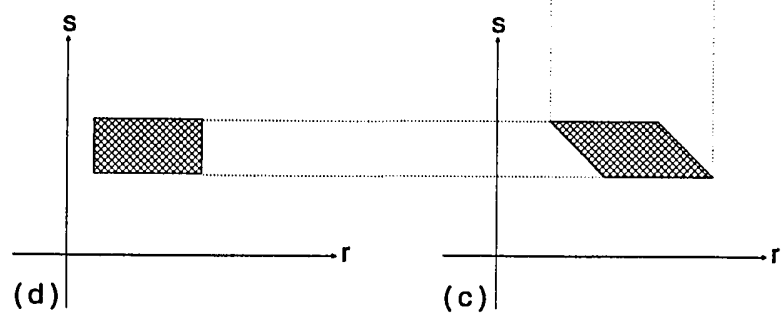

Fig. 4. Phase-space representation of the original bundle of rays (a), after free-space propagation through a distance $f(b)$, after passage through a lens of focal length $f$ (c), and after another free-space propagation through a distance $f(\mathrm{~d})$.

Let us now consider a bundle of rays with a uniform spread of $r$ and $s$ [represented by the crosshatched rectangular region in Fig. 4(a)] and consider how this ray bundle is transformed as it passes through a conventional $2 f$ Fourier-transforming configuration (Fig. 4). The overall effect of the $2 f$ system is to rotate the rectangular region by $90^{\circ}$, although the intermediate steps result in shearing of the rectangular region.

We now examine the effect of fractional Fourier transforming in GRIN media on the rectangular region in Fig. 4(a). It is known that $r$ and $s$ obey the following equations in such media ${ }^{15}$ :

$$
\begin{aligned}
& r(z+\Delta z)=r(z) \cos (\pi \Delta z / 2 L)-s(z) \sin (\pi \Delta z / 2 L), \\
& s(z+\Delta z)=r(z) \sin (\pi \Delta z / 2 L)+s(z) \cos (\pi \Delta z / 2 L),
\end{aligned}
$$

from which we can conclude that the region representing any given bundle of rays in phase space is uniformly rotated as $z$ is increased. An increment of $\Delta z=L$, a full Fourier-transform distance, corresponds to a rotation by $90^{\circ}$. This uniform behavior is to be contrasted with that of the bulk $2 f$ Fourier transformer, in which the focusing is concentrated at the lens instead of being uniformly distributed throughout the system. For this reason it is not attractive to define fractional Fourier transforms based on conventional Fourier-transforming systems.

\section{CONCLUSION}

We have defined the fractional Fourier-transform operator $\mathscr{F}^{a}$ such that the common Fourier-transform operator is a special case with $a=1$. Our optically motivated definition was seen to be identical to a purely mathematical derivation found in the mathematics literature. We discussed several properties of this transform, in particular showing that they can be realized with quadratic graded index (GRIN) media. Although the definition of the fractional Fourier transform is not unique, our definition is useful from the physical optical point of view. The two postulates on which the definition is based are well matched to a very useful optical device-the GRIN medium.

Fractional Fourier transforms can form the basis of generalized spatial filtering operations, extending the range of operations possible with optical information processing systems. Conventional Fourier plane filtering systems ${ }^{13}$ are based on a spatial filter introduced at the Fourier plane, which limits the achievable operations to space-invariant ones. By introducing several filters at different fractional Fourier planes, one can implement a wider class of operations.

It is important to note that, although they provide a context for defining fractional Fourier transforms in a satisfying manner, GRIN media may not be good candidates for practical implementation, especially because they cannot have large space-bandwidth products. For practical purposes, it is possible to simulate GRIN media by the use of bulk lenses such that the same light distribution will be observed at the planes in which filtering will take place. ${ }^{10}$

Several of the issues mentioned above will be taken up in the sequel to this paper. ${ }^{10}$ In addition, we will provide illustrative examples of fractional Fourier transforms and introduce some extensions and generalizations.

\section{ACKNOWLEDGMENTS}

Some of the ideas in this paper were developed during intensive study sessions organized by Adolf W. Lohmann at the Applied Optics Group of the University of ErlangenNürnberg during the summer of 1992. The authors acknowledge the useful suggestions of Klaus Leeb of the Informatik Department of the University of Erlangen.

\section{REFERENCES}

1. R. Bracewell, The Fourier Transform and Its Applications, 2nd ed. (McGraw-Hill, New York, 1986).

2. A. Zygmund, Trigonometric Series, 1 (Cambridge U. Press, Cambridge, 1979).

3. P. I. Lizorkin, "Fractional integration and differentiation," in Encyclopedia of Mathematics (Kluwer, Dordrecht, The Netherlands), Vol. 4.

4. A. Vander Lugt, Optical Signal Processing (Wiley, New York, 1992).

5. A. Lohmann and D. Mendlovic, "Image formation and selfFourier object," submitted to Appl. Opt.

6. K. Patorski, "The self-imaging phenomenon and its applications," in Progress in Optics, E. Wolf, ed. (North-Holland, Amsterdam, 1989), Vol. 28, p. 3-110.

7. A. Lohmann and D. Mendlovic, "Self-Fourier objects and other self-transform objects," J. Opt. Soc. Am. A 9, 20092012 (1992).

8. A. Lohmann and D. Mendlovic, "Optical self-transform with odd order," Opt. Commun. 93, 25-26 (1992).

9. H. Ozaktas and D. Mendlovic, "Fourier transforms of fractional order and their optical interpretation," Opt. Commun. (to be published).

10. H. Ozaktas and D. Mendlovic, "Fractional Fourier transforms and their optical implementation: II," J. Opt. Soc. Am. A (to be published).

11. V. Namias, "The fractional Fourier transform and its application in quantum mechanics," J. Inst. Math. Its Appl. 25, 241 265 (1980).

12. A. C. McBride and F. H. Kerr, "On Namias's fractional Fourier transforms," IMA J. Appl. Math. 39, 159-175 (1987).

13. J. Goodman, Introduction to Fourier Optics (McGraw-Hill, New York, 1968). 
14. A. Lohmann, "Ein neues Dualitatsprinzip in der Optik," Optik (Stuttgart) 11, 478-488 (1954). An English version appeared in Optik (Stuttgart) 89, 93-97 (1992).

15. A. Yariv, Optical Electronics, 3rd ed. (Holt Reinhart, New York, 1985).

16. A. Yariv, Quantum Electronics (Wiley, London, 1975).
17. A. Erdelyi, Tables of Integral Transforms (McGraw-Hill, New York, 1954), Vol. 1, p. 369.

18. B. E. A. Saleh and M. C. Teich, Fundamental of Photonics (Wiley, New York, 1991).

19. M. Abramovitz and I. A. Stegun, Handbook of Mathematical Functions (Dover, New York, 1970). 